\title{
Variação da viabilidade polínica em Tibouchina (Melastomataceae)
}

\author{
Variation of pollen viability in Tibouchina (Melastomataceae)
}

\author{
Glaucia Margery Hoffmann ${ }^{1}$ \& Isabela Galarda Varassin ${ }^{2}$
}

\begin{abstract}
Resumo
O gênero Tibouchina é comum no Brasil e encontrado principalmente na Floresta Atlântica, em especial na região Sudeste. No presente estudo foi avaliada a viabilidade polínica de cinco espécies de Floresta Atlântica, T. cerastifolia, T. clinopodifolia, T. gracilis, T. pulchra e T. sellowiana, utilizando-se três soluções histoquímicas: carmim acético, Alexander e cloreto de 2,3,5-trifenil-tetrazólio. Foi avaliada a variação de viabilidade entre as anteras dimórficas, entre as soluções histoquímicas e entre os indivíduos. Em T. pulchra e T. sellowiana também foi avaliada a variação entre os dois primeiros dias a partir da abertura da flor. Nenhuma espécie apresentou variação relacionada ao dimorfismo das anteras. Nas duas espécies que apresentam maior longevidade floral, a viabilidade foi semelhante no primeiro e segundo dias em T. pulchra, enquanto que em T. sellowiana as flores de segundo dia apresentaram pólen menos viável. As soluções histoquímicas empregadas revelaram taxas de viabilidade polínica distintas. Houve variação na viabilidade polínica entre os indivíduos em todas as espécies. A solução de cloreto de 2,3,5-trifenil-tetrazólio revelou a menor taxa de viabilidade em todos os indivíduos. As soluções histoquímicas empregadas, a amostragem e a longevidade floral foram fatores relacionados à variação da viabilidade polínica.
\end{abstract}

Palavras-chave: pólen, polinização, reprodução, soluções histoquímicas.

\begin{abstract}
The genus Tibouchina is common in Brazil, mainly found in Atlantic Forest, in Southeast region. In this study the pollen viability was evaluated in five species of Atlantic Forest, T. cerastifolia, T. clinopodifolia, T. gracilis, T. pulchra and T. sellowiana, using three histochemical solutions: acetocarmine, Alexander and 2, 3, 5-triphenyltetrazolium chloride. The variation of pollen viability was evaluated among the dimorphics anthers and among individuals. In T. pulchra and T. sellowiana it was also evaluated the variation among the two first days after flower openning. There was no variation related to anther dimorphism. Considering the two species with the longest flower longevity, the viability was similar in the first and second day in T. pulchra, but in T. sellowiana the pollen from the second day was less viable. The histochemical tests employed showed distinct rates of pollen viability. Rates of pollen viability were distinct among individuals in each species. The stain 2, 3, 5-triphenyltetrazolium chloride showed the smaller viability. The results show that the treatments employed, sampling and flower longevity were factors related to pollen viability.
\end{abstract}

Key words: histochemical solutions, pollen, pollination, reproduction.

\section{Introdução}

As flores de Tibouchina Aubl. (Melastomataceae) são quase que exclusivamente polinizadas por abelhas (Renner 1989) sendo bastante atrativas, tanto pela coloração de suas pétalas quanto pela produção de pólen. As abelhas recolhem o pólen das anteras poricidas de formato tubuloso por meio de vibrações, chamada de polinização por vibração (Renner 1989; Larson \&
Barrett 1999; Goldenberg \& Varassin 2001). Estas vibrações proporcionam a deposição de pólen no abdômen e nas laterais do corpo da abelha (Renner 1989; Larson \& Barrett 1999). Os grãos de pólen coletados são levados para os ninhos das abelhas para alimentação das larvas (Renner 1989; Schlindwein et al. 2005).

Em muitas espécies de Melastomataceae, como as do gênero Tibouchina, há existência de flores com estames dimórficos (heteranteria sensu

${ }^{1}$ Universidade Tuiuti do Paraná, R. Sydnei Antônio Rangel Santos 238, 82010-330,Santo Inácio, PR, Brasil.

${ }^{2}$ UFPR, Centro Politécnico, Setor de Ciências Biológicas, Dep. Botânica, C.P. 19031, 81531-980, Curitiba, PR, Brasil. Autor para correspondência: isagalarda@ufpr.br 
Endress 1994). O fato de haver estames maiores e menores faz com que, durante a visita às flores, abelhas de grande porte abracem todas as anteras, enquanto que as abelhas menores abracem as anteras uma a uma (Renner 1989; Oliveira-Rebouças \& Gimenes 2004). A heteranteria tem sido interpretada como uma divisão funcional de trabalho entre as anteras (Faegri \& van der Pilj 1979; Buchmann 1983), devido a uma deposição diferencial do pólen de anteras de ciclos diferentes em regiões distintas do corpo das abelhas. Existem anteras funcionais, de fertilização, cujo pólen não é retirado do corpo das abelhas durante a limpeza e, portanto, estão envolvidos no processo de polinização. Outro tipo de anteras são as de alimento, cujo pólen é coletado para alimentação (Renner 1989). Associado a isto também foi considerado que apenas nos estames longos, os de fertilização, os grãos de pólen são férteis (Renner 1989). Em espécies que exibem heteranteria, a implicação de diversos tipos de anteras para reprodução tem sido investigada. Em Solanum L. (Solanaceae) a presença de mais de um tipo de antera não implica em diferenças reprodutivas para a planta (Bowers 1975), mas em Melastoma malabathricum L. (Melastomataceae), Luo et al. (2008) observaram uma maior deposição estigmática de grãos de pólen oriundos de anteras maiores. Em função destas diferenças, é possível que a viabilidade polínica seja distinta em anteras maiores e menores.

Ainda que os polinizadores contribuam significativamente para a reprodução em Tibouchina, outros fatores ligados à biologia do grão de pólen podem afetar a reprodução. A viabilidade e a longevidade polínicas, ou mesmo sua capacidade de fertilização, podem ser afetadas, por exemplo, pela ocorrência de dessecação, pela baixa umidade relativa, disponibilidade de substância de reserva, idade da flor, metabolismo, alterações morfológicas da antera, temperatura entre outros (Dafni \& Firmage 2000).

A fim de compreender a biologia da polinização são também necessários estudos que visem avaliar a fertilidade do grão de pólen e os fatores que podem alterar a viabilidade. É possível avaliar ou estimar a fertilidade masculina a partir de testes simples, como testes histoquímicos, focando na viabilidade polínica (Stanley 1965; Kearns \& Inouye 1993; Dafni \& Firmage 2000). Existem diversos métodos para estimar a viabilidade polínica, havendo vantagens e desvantagens no uso de cada um deles (Dafni \& Firmage 2000).
Dentre os métodos histoquímicos, três soluções são frequentemente usadas, sendo elas a solução de carmim acético, solução de Alexander e solução de cloreto de 2,3,5-trifenil-tetrazólio (TTC).

$\mathrm{O}$ presente artigo tem por objetivo avaliar a variação da viabilidade polínica em cinco espécies do gênero Tibouchina, sendo duas espécies arbóreas (T. pulchra Cogn. e $T$. sellowiana Cogn.) e três espécies arbustivas ( $T$. cerastifolia Cogn., $T$. clinopodifolia Cogn. e T. gracilis (Bonpl.) Cogn. Para isso, foram considerados o dimorfismo das anteras (diferença de viabilidade polínica das anteras maiores e menores), a possível perda de viabilidade durante a antese nas espécies arbóreas e a relevância das soluções histoquímicas para a realização de testes de viabilidade.

\section{Material e Métodos}

Foram coletados botões e flores de cinco indivíduos de cada uma das espécies de Tibouchina, sendo T. cerastifolia, T. gracilis, T. pulchra e T. sellowiana os indivíduos coletados em área urbana de Curitiba, PR e T. clinopodifolia nos mananciais da Serra, Piraquara, PR (Reserva da SANEPAR). Os materiais foram depositados no Herbário UPCB (I. G. Varassin 173; I. G. Varassin 174; I. G. Varassin 175; I. G. Varassin 176; I. G. Varassin 177).

Nas espécies arbustivas, T. clinopodifolia, T. cerastifolia, T. gracilis, as flores foram coletadas logo após a abertura das flores, pela manhã. Para as espécies arbóreas, T. pulchra e T. sellowiana, foram coletadas flores de primeiro dia (flores brancas) e flores de segundo dia (mudando coloração para o rosa), também no período da manhã. A viabilidade das anteras maiores e menores foi avaliada separadamente para cada indivíduo para avaliar um possível papel diferencial dos dois tipos de antera na reprodução. Em T. pulchra e T. sellowiana foi comparada a influência da duração da antese na viabilidade dos grãos de pólen.

Os grãos de pólen foram extraídos com o auxílio de pinça, sendo dispostos em lâmina e submetidos aos testes histoquímicos com três corantes específicos separadamente: solução de Alexander (Alexander 1980), solução de Carmim Acético (Kearns \& Inouye 1993) e solução de cloreto de 2,3,5-trifenil tetrazólio 0,5\% (Cook \& Stanley 1960). A solução de Alexander cora o protoplasma (Alexander 1980; Dafni 1992) e distingue os grãos de pólen abortados dos não abortados. O composto verde malaquita reage com a celulose da parede celular do pólen e a fucsina ácida com o 
protoplasma, refletindo a coloração púrpura (Alexander 1980; Kearns \& Inouye 1993). Para essa solução foram considerados viáveis grãos corados de púrpura, enquanto que os não viáveis corados de verde (pólen abortado, deficiente em protoplasma) (Alexander 1980). A solução de carmim acético atua sobre os cromossomos do grão de pólen não abortado, corando-o, e podendo corar ainda pólen imaturo e inviável (Stanley \& Linskens 1974; Kearns \& Inouye 1993). Para essa solução, foram considerados viáveis os grãos de pólen corados de vermelho e não viáveis os grãos não corados. A solução de 2,3,5-trifenil-tetrazólio ou TTC é distinta dos outros testes por envolver uma reação enzimática (Stanley 1965; Stanley \& Linskens 1974; Kearns \& Inouye 1993). Os sais de cloreto de 2,3,5-trifeniltetrazólio confirmam a viabilidade polínica através das enzimas ativas que provocam mudanças na cor dos grãos para o vermelho na presença da atividade oxidativa. A reação do corante indica a presença da atividade enzimática contida nas células vivas (Vieitez 1952; Stanley 1965). Para essa solução foram considerados viáveis os grãos de pólen que coraram de vermelho e não viáveis os sem coloração ou muito claros.

Foram avaliadas 210 lâminas em campos aleatórios com aumento de 10x, considerando 100 grãos de pólen por lâmina. Em cada solução histoquímica empregada foi definido o percentual de grãos viáveis. A diferença de viabilidade média entre as anteras dimórficas em cada espécie, assim como entre flores de primeiro e segundo dia de antese foram avaliadas por teste $t$. As diferenças de viabilidade polínica média entre os indivíduos e os tratamentos empregados foram avaliadas por análise de variância de dois fatores ou unifatorial. Para os resultados que não apresentaram influência de uma variável sobre a outra, as médias foram então analisadas separadamente (Zar 1996). Um dos indivíduos de T. gracilis apresentava as anteras menores predadas, acarretando em resultados negativos no tratamento com TTC e, portanto, este dado foi excluído para não interferir nos demais resultados.

\section{Resultados}

Em relação ao dimorfismo das anteras, tanto as grandes quanto as pequenas apresentaram a mesma viabilidade polínica em todas as espécies (Fig. 1a), T. cerastifolia $\left(t_{28}=0,36 ; P>0,05\right), T$. clinopodifolia $\left(\mathrm{t}_{28}=0,46 ; P>0,05\right)$, . . gracilis $\left(t_{27}=-\right.$ $0,19 ; P>0,05), T$. pulchra $\left(t_{58}=-0,07 ; P>0,05\right), T$ sellowiana $\left(t_{58}=-0,46 ; P>0,05\right)$.
Em relação ao período de antese, em T. pulchra as flores de primeiro dia e segundo dia apresentaram a mesma viabilidade $\left(t_{58}=1,03 ; P>0,05\right.$; Fig. $\left.1 \mathrm{~b}\right)$ enquanto que em $T$. sellowiana, as flores de segundo dia apresentaram menor viabilidade $\left(r^{2}=0,60 ; \mathrm{F}_{1: 54}=14,47 ; P<0,05\right)$. A resposta entre os tratamentos variou durante a antese $\left(r^{2}=0,60\right.$; $\left.\mathrm{F}_{2: 54}=4,48 ; P<0,05\right)$.

De uma maneira geral, os tratamento histoquímicos empregados revelaram viabilidades polínicas distintas (Fig. 1c). A viabilidade polínica foi consistentemente menor nos ensaios onde se usou solução de TTC e foi semelhante entre os tratamentos usando a solução de Alexander e solução de Carmim Acético (Fig. 1c).

Em todas as espécies, à exceção de $T$. sellowiana, a viabilidade polínica variou entre os indivíduos, assim como os tratamentos empregados revelaram taxas distintas de viabilidade (Tab. 1), sendo para algumas espécies, como T. clinopodifolia, T. gracilis, T. pulchra, os indivíduos responderam de forma distinta para os três tratamentos (Tab. 1).

\section{Discussão}

Apesar da possibilidade de haver divisão de atividade entre as anteras dimórficas em Melastomataceae (Renner 1989; Buchmann 1983; Luo et al. 2008), não foi constatada variação na viabilidade polínica entre os diferentes tipos de anteras das espécies aqui estudadas. Esta ausência de variação na viabilidade polínica nas anteras dimórficas foi também constatada em Cambessedesia hilariana (Fracasso \& Sazima 2004) e em T. pulchra (Silva 2006). Esta similaridade na viabilidade polínica em ambos os tipos de anteras deve ser em função do processo meiótico, que é o mesmo em ambas as anteras, independentemente de seu papel (na reprodução ou como fonte alimentar para abelhas). Deste modo, nas espécies de Tibouchina estudadas, os grãos de pólen oriundos dos dois tipos de anteras poderiam ser usados para reprodução.

Tibouchina pulchra e T. sellowiana, apresentam, entre elas, uma diferença na queda da viabilidade desde a antese até o fenecimento da flor. A viabilidade mais longa em T. pulchra pode estar associada com a manutenção da funcionalidade reprodutiva da flor, uma vez que a flor está disponível aos polinizadores por até nove dias (Silva 2006). Em T. sellowiana, como cada flor dura de dois a três dias (Goldenberg \& Varassin 2001), uma extensão 

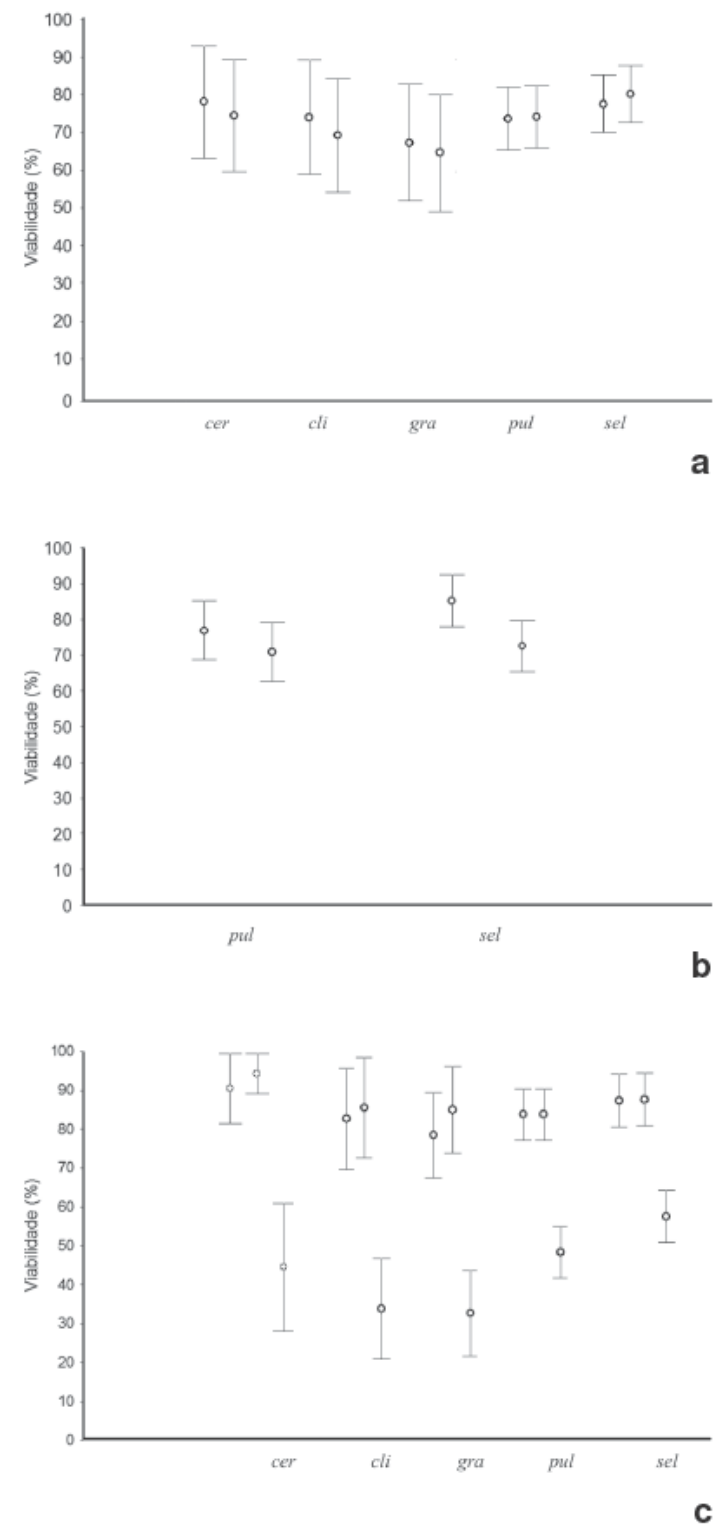

Figura 1 - Viabilidade polínica em Tibouchina - a. em anteras maiores (esquerda) e menores (direita); b. em flores de primeiro (esquerda) e segundo dia de antese (direita); c. em diferentes tratamentos histoquímicos: Alexander (esquerda), Carmim acético (centro) e TTC $0,5 \%$ (direita). cer $=T$. cerastifolia; $c l i=$ $T$. clinopodifolia; gra $=T$. gracilis; pul $=T$. pulchra; sel $=T$. sellowiana

Figure 1 - Pollen viability in Tibouchina - a. in the larger anthers (left) and smaller (left); b. in flowers from the first (left) and second day of anthesis (right); c. in different histochemical solutions: Alexander (left), Acetic-carmine (center) and TTC $0,5 \%$ (right). da viabilidade polínica por mais tempo não resultaria em maior chance de fecundação. Isto pode também estar relacionado com o período de visitação das abelhas. Enquanto em T. sellowiana os polinizadores visitam flores apenas no dia em que a flor abriu (Goldenberg \& Varassin 2001), em T. pulchra podem ocorrer visitas no segundo dia, porém em menor proporção (Silva 2006). Neste caso, visitas posteriores ao primeiro dia podem ainda resultar em fecundação já que, do ponto de vista masculino, a viabilidade é mantida. Ensaios realizados por Silva (2006) em T. pulchra demonstram que os grãos de pólen de segundo dia são capazes de fecundar a oosfera com formação de frutos similar aos ensaios com grãos de pólen de flores de primeiro dia. Silva (2006) aponta ainda em seu estudo, a influência da diminuição da viabilidade polínica na formação de frutos com os grãos de pólen provenientes de flores de terceiro dia. A redução de viabilidade polínica em $T$. sellowiana no segundo dia de antese foi observada por Goldenberg e Varassin (2001) e se reflete em redução do sucesso reprodutivo, uma vez que polinizações em flores de segundo dia têm menor sucesso do que as de primeiro dia (Goldenberg \& Varassin 2001). Em autopolinizações espontâneas os tubos polínicos conseguiram atingir os óvulos, mas em apenas $3,4 \%$ do total de ensaios ocorreu fecundação da oosfera e formação de frutos (Goldenberg \& Varassin 2001).

$\mathrm{O}$ fato dos tratamentos histoquímicos empregados revelarem diferentes respostas de viabilidade era esperada, pois cada teste histoquímico atua sobre um componente do grão de pólen. Comparando os três testes histoquímicos, é interessante ressaltar que a viabilidade média foi mais baixa em todas as espécies e indivíduos com os testes conduzidos com a solução de 2,3,5trifenil-tetrazólio. Isto parece indicar que mesmo grãos de pólen íntegros e com cromossomos viáveis podem ter uma redução na viabilidade em função de baixa atividade enzimática dos grãos de pólen, uma vez que o TTC atua sobre as enzimas desidrogenases e peroxidases ativas, relacionadas com a respiração celular (Stanley 1965; Stanley \& Linskens 1974; Kearns \& Inouye 1993).

A alta viabilidade polínica encontrada aqui para todas as espécies pode estar associada à ausência de apomixia e ocorrência de autocompatibilidade (Goldenberg \& Varassin 2001), conforme observado em $T$. cerastifolia, $T$. clinopodifolia, $T$. gracilis (Franco 2007), T. pulchra (Silva 2006)e T. sellowiana 
Tabela 1 - Variação da viabilidade polínica entre os indivíduos, entre os tratamentos e da interação entre indivíduo e tratamento (Anova uni- ou bifatorial).

Tabela 1 - Variation of pollen viability among individuals, treatments, and interaction among individuals and treatments (One- or twoway Anova).

\begin{tabular}{|c|c|c|c|}
\hline Espécie & $\begin{array}{l}\text { Variação } \\
\text { individual }\end{array}$ & $\begin{array}{l}\text { Variação entre } \\
\text { tratamentos }\end{array}$ & $\begin{array}{l}\text { Efeito do indivíduo } \\
\text { sobre o tratamento }\end{array}$ \\
\hline Tibouchina cerastifolia & $\begin{array}{l}r^{2}=-0,06 \\
\mathrm{~F}_{4,25}=0,62 \\
P<0,05\end{array}$ & $\begin{array}{l}r^{2}=-0,68 \\
\mathrm{~F}_{2,27}=31 \\
P<0,05)\end{array}$ & ns \\
\hline Tibouchina clinopodifolia & $\begin{array}{l}r^{2}=0,94 \\
\mathrm{~F}_{4,15}=34,07 \\
P^{\circ}<0,05\end{array}$ & $\begin{array}{l}r^{2}=0,94 \\
\mathrm{~F}_{2: 15}=153,03 \\
P<0,05\end{array}$ & $\begin{array}{l}r^{2}=0,94 \\
\mathrm{~F}_{8: 15}=5,39 \\
P<0,05\end{array}$ \\
\hline Tibouchina gracilis & $\begin{array}{l}r^{2}=0,92 \\
\mathrm{~F}_{4 ; 14}=13,16 \\
P<0,05\end{array}$ & $\begin{array}{l}r^{2}=0,92 \\
\mathrm{~F}_{2 ; 14}=104,86 \\
P<0,05\end{array}$ & $\begin{array}{l}r^{2}=0,92 \\
\mathrm{~F}_{8 ; 14}=5,62 \\
P<0,05\end{array}$ \\
\hline Tibouchina pulchra & $\begin{array}{l}r^{2}=0,84 \\
\mathrm{~F}_{4 ; 45}=22,00 \\
P<0,05\end{array}$ & $\begin{array}{l}r^{2}=0,84 \\
\mathrm{~F}_{22 ; 5}=104,14 \\
P<0,5\end{array}$ & $\begin{array}{l}r^{2}=0,84 \\
\mathrm{~F}_{8 ; 45}=2,50 \\
P<0,05\end{array}$ \\
\hline Tibouchina sellowiana & $\begin{array}{l}r^{2}=0,05 \\
\mathrm{~F}_{4,55}=1,84 \\
P>0,05\end{array}$ & $\begin{array}{l}r^{2}=0,05 \\
\mathrm{~F}_{4 ; 55}=1,84 \\
P>0,05\end{array}$ & ns \\
\hline
\end{tabular}

(Goldenberg \& Varassin 2001). Espécies do gênero Leandra Raddi e Miconia Ruiz \& Pav. possuem baixas taxas de viabilidade polínica (Goldenberg 2000), geralmente em função de malformações polínicas de indivíduos de origem poliplóide. De fato, em algumas espécies apomíticas de Melastomataceae foram observadas irregularidades meióticas, provavelmente responsáveis pelos baixos níveis de viabilidade polínica (Goldenberg \& Shepherd 1998).

A variação individual da viabilidade polínica demonstra a importância de estudos populacionais para avaliar a fertilidade masculina. Neste estudo, na maioria das espécies trabalhadas, os indivíduos de uma mesma espécie apresentaram viabilidade polínica variável.

As diferenças de viabilidade encontradas neste estudo indicam que a estimativa de viabilidade polínica pode ser afetada pela amostragem (indivíduo) e que o uso de soluções histoquímicas diferentes influi nos resultados encontrados de viabilidade. Além disso, fatores biológicos e ecológicos como a longevidade da flor podem estar associados à variação na taxa de viabilidade polínica. O dimorfismo das anteras, por outro lado, não se reflete em diferenças de viabilidade polínica para essas espécies de Tibouchina.

\section{Agradecimentos}

À Miriam Kaehler pelas sugestões, à UFPR por disponibilizar os laboratórios, à SANEPAR pela autorização das coletas e à EMBRAPA FLORESTAS e UTP pelos reagentes. Esse estudo faz parte do Trabalho de conclusão de curso da primeira autora.

\section{Referências}

Alexander, M.P. 1980. A versatile stain for pollen fungi, yeast and bacteria. Stain Technology 55: 13-18.

Bowers, K.A.W. 1975. The pollination of Solanum rostratum (Solanaceae). American Journal of Botany 62: 633-638.

Buchmann, S.L. 1983. Buzz pollination in Angiosperms. In: Jones, C.E. \& Little, R. J. (eds.). Handbook of experimental pollination biology. Van Nostrand \& Reinhold, New York. Pp. 73-113.

Cook, S.A. \& Stanley, R.G. 1960. Tetrazolium chloride as indicator of pine pollen germinability. Silvae Genetic 9: 134-136.

Dafni, A. 1992. Pollen and stigma biology. In: Pollination ecology: a practical approach. Oxford University Press, Oxford. Pp. 59-89.

Dafni, A. \& Firmage, D. 2000. Pollen viability and longevity: practical, ecological and evolutionary implications. Plant Systematics and Evolution 222: 113-132.

Endress, P.K. 1994. Diversity and evolutionary biology of tropical flowers.Cambridge University Press, Cambridge. 530p. 
Faegri, K. \& van der Pijl, L. 1979. The principles of pollinations ecology. 3ed. Pergamon, New York. $291 \mathrm{p}$.

Fracasso, C.M \& Sazima, M. 2004. Polinização de Cambessedesia hilariana (Kunth) DC (Melastomataceae): sucesso reprodutivo versus diversidade, comportamento e frequência de visitas de abelhas. Revista Brasileira de Botânica 27: 797-804.

Franco, A.M. 2007. Biologia reprodutiva de três espécies de Melastomataceae (Tibouchina cerastifolia Cogn., T. clinopodifolia Cogn. e T. gracilis Cogn.) nos Mananciais da Serra, Piraquara, Paraná. Dissertação de Mestrado. Universidade Federal do Paraná, Curitiba. 57p.

Goldenberg, R. 2000. Apomixia como alternativa à reprodução em Melastomataceae. In: Cavalcanti, T. B \& Walter, B.M.T. Tópicos Anuais em Botânica. Embrapa Recursos Genéticos Brasília. Pp. 225-230.

Goldenberg, R. \& Shepherd, G.J. 1998. Studies on the reproductive biology of Melastomataceae in cerrado vegetation. Plant Systematics and Evolution 211: 13-29.

Goldenberg, R. \& Varassin, I.G. 2001. Sistemas reprodutivos de espécies de Melastomataceae da Serra do Japi, Jundiaí, São Paulo, Brasil. Revista Brasileira Botânica 24: 283-288.

Kearns, C.A. \& Inouye, D.W. 1993. Pollen. In: Techniques for pollination biologist. Colorado University Press, Niwot. Pp. 77-151.

Larson, B.M.H. \& Barrett, S.C.H. 1999. The pollination ecology of buzz pollinated Rhexia virginica (Melastomataceae). American Journal of Botany 86: 502-511.
Luo, Z.; Zhang, D \& Renner, S. S. 2008. Why two kinds of stamens in buzz-pollinated flowers? Experimental support for Darwin's division-of-labour hypothesis. Functional Ecology 22: 794-800.

Oliveira-Rebouças, P. \& Gimenes, M. 2004. Abelhas (Apoidea) visitantes de flores de Comolia ovalifolia DC Triana (Melastomataceae) em uma área de restinga na Bahia. Neotropical Entomology 33: 315-320.

Renner, S.S. 1989. A survey of reproductive biology in neotropical Melastomataceae and Memecylaceae. Annals of the Missouri Botanical Garden 76: 496-518.

Schlindwein, C.; Wittmann, D.; Martins, C.F.; Hamm, A.; Siqueira, J.A.; Schiffler, D. \& Machado, I.C. 2005. Pollination of Campanula rapunculus $\mathrm{L}$. (Campanulaceae): how much pollen flows into pollination and into reproduction of oligolectic pollinators? Plant Systematics and Evolution. 250: 147-156.

Silva, J.B. 2006. Biologia das interações entre os visitantes florais (Hymenoptera, Apidae) em Tibouchina pulchra Cogn. (Melastomateceae). Dissertação de Mestrado. Universidade Federal do Paraná, Curitiba. 62p.

Stanley, R.G. 1965. Physiology and uses of tree pollen. Agricultural Science Review 3: 9-17.

Stanley, R.G \& Linskens, H. 1974. Viability tests. In: Pollen: biology, biochemistry, management. Springer-Verlag, Berlin. Pp. 67-86.

Vieitez, E. 1952. El uso del Cloruro 2,3,5trifeniltetrazolium para determinar la vitalidad del polen. Anales de Edafología y Fisiologia Vegetal 11: 297-308.

Zar, J.H. 1996. Biostatistical analysis. 3 ed. Prentice Hall, New Jersey. 718p. 